\title{
Niñez y Derechos Humanos: entre el desamparo y la justicia
}

\author{
Martín Menestrina, Carolina Borrego, \\ Lucia Belaunzaran, Sebastián Pagano, \\ Martina Flaherty y Lucía Zapico
}

Universidad

Nacional de

La Plata

Revista Derechos en Acción

Año 3/Nº 9 Primavera 2018, 527-539

DOI: https://doi.org/10.24215/25251678e240

ORCID: https://orcid.org/0000-000

Resumen: El Programa "Niñez, Derechos Humanos y Políticas Públicas" de la Secretaría de Extensión de la Facultad de Ciencias Jurídicas y Sociales (UNLP) surgió a raíz de una experiencia territorial colectiva de denuncia y exigibilidad al Estado del cumplimiento de sus obligaciones legales respecto a la niñez íntimamente vinculado a la violenta represión que sufrieron un grupo de niños y niñas de entre 6 y 17 años que vivían en la plaza San Martín de la ciudad de La Plata en el año 2008. Estos hechos pusieron de manifiesto que la Provincia de Buenos Aires y la Municipalidad de La Plata estaban incumpliendo con las obligaciones asumidas en la ley 13.298 "Sistema de Promoción y Protección de los Derechos del Niño" que había establecido un cambio de paradigmas: del sistema tutelar al sistema de la protección integral. En ese contexto se presentó una demanda judicial solicitando la efectiva puesta en funcionamiento de la ley 13.298. En el presente artículo abordamos algunas de las cuestiones fácticas y jurídicas más relevantes de ese proceso que ha durado más de 10 años, y desde hace 6 años espera una sentencia de la Suprema Corte de Justicia de la Provincia de Buenos Aires.

Summary: The "Children, Human Rights and Public Policies" Program of the Extension Secretariat of the Faculty of Legal and Social Sciences (UNLP) arose as a result of a collective territorial experience of demand to the government for the fulfillment of its legal obligations due to the repression that suffered a group of children who lived in the Plaza San Martín in the city of La Plata in 2008. These facts revealed that the local 
government fails to comply with the obligations assumed in law 13.298 "Promotion and Protection of the Rights of the Child" It had established a paradigm shift: from a tutelary to a comprehensive protection. In this context, a lawsuit was filed to force the government to comply with Law 13.298. In the present article we address some of the most relevant factual and legal aspects of this process that has lasted more than 10 years, and for 6 years awaiting a ruling from the Supreme Court of Justice of the Province of Buenos Aires.

\section{De frazadas y ollas}

En el año 2008 un grupo de 15 niños y niñas de entre 6 y 17 años que se encontraba en situación de calle y que circulaban por la Facultad de Humanidades -en aquel momento funcionaba en el edificio de calle 48 entre 6 y 7 - fue expulsado por sus autoridades y se trasladó a la glorieta de Plaza San Martín. Los medios de comunicación los habían estigmatizado como " $\mathrm{La}$ banda de la frazada" (diario El Día, 28/7/2008). Las crónicas de aquel tiempo dan cuenta de que los chicos de la plaza San Martín tiraban una frazada sobre los transeúntes para sorprenderlos y luego robarles. Los niños se quedaban con los celulares que después cambiaban en las ferreterías por pegamento, o por sándwiches en un kiosco próximo a la Comisaría Segunda de la capital provincial.

Para ese entonces un grupo de estudiantes de la carrera de Trabajo Social, talleristas y operadores de calle que venían trabajando con esos niños y niñas desde la Dirección de Niñez de la Municipalidad de La Plata, pero que habían abandonado dicho espacio debido a la ausencia de recursos materiales para trabajar y a las condiciones de precariedad laboral, conformaron un grupo denominado "Autoconvocados por los derechos de los pibes en situación de calle". El mismo se fue integrando por Organizaciones Sociales y de Derechos Humanos, instituciones académicas y personas interesadas en la problemática.

Entre las intervenciones desarrolladas por aquel grupo de personas se encontraba la realización de una olla popular 
nocturna cuyo objeto era realizar acciones para contener a los niños que se encontraban viviendo en la plaza.

Luego de unos días desde que comenzó la olla, el 25 de julio casi a la media noche cuando el grupo se retiraba y ya se habían apagado todas las luces de la plaza, más de 20 adultos, mujeres y hombres, vestidos de civil algunos y otros identificándose como policías, armados con palos, cadenas y armas de fuego llegaron y golpearon y reprimieron a los niños bajo la consigna de "limpiar" la plaza. Sobre una de las calles que daba a la plaza, había cuatro agentes de la policía (que vigilaban a la olla popular desde su primer día) que en lugar de impedir la agresión, intentaron retener a los chicos que querían escapar.

A partir de ese hecho el grupo de autoconvocados, que pasó a llamarse "Asamblea Permanente por los derechos de la niñez”, entendió la necesidad de ampliar el espacio de reflexión y lucha, para lo cual se denunció penalmente lo sucedido así como también se realizaron diversas presentaciones ante los organismos administrativos de niñez, sin que se hayan obtenido respuestas en favor de estos niños, evidenciando la ausencia de programas y políticas públicas en materia de niñez.

Frente a la paradoja de que el Estado criminalizaba a estos niños por hechos que tenían su origen en la omisión de haber implementado acciones integrales e inclusivas de derechos humanos, un grupo de madres, organizaciones sociales e instituciones académicas promovieron una acción de amparo -con el patrocino de algunos miembros del Programa "Niñez, Derechos Humanos y Políticas Públicas"- contra la Provincia de Buenos Aires y la Municipalidad de La Plata solicitando la efectiva puesta en marcha de la ley 13.298 que había creado el Sistema de Promoción y Protección de los Derechos del Niño (SPPDN)

\section{Un nuevo paradigma}

A partir de la incorporación de la Convención sobre los Derechos del Niño al sistema jurídico nacional en 1990, con 
jerarquía constitucional desde 1994, y a la preocupación expresada por el Comité de los Derechos del Niño sobre la legislación provincial basada en la doctrina de la "situación irregular" (Comité Derechos del Niño, 2002), la Provincia de Buenos Aires sancionó en el año 2005 la Ley 13.298 de Promoción y Protección Integral de los Derechos de los Niños, que asimismo derogó el decreto-ley 10.067/83 "Ley de patronato provincial".

El eje fundamental es el reconocimiento de los niños, niñas y jóvenes como sujetos de derechos que, junto con el establecimiento de su derecho a ser escuchados, constituyó un cambio de paradigma respecto del viejo modelo del patronato.

Aquél antiguo sistema respondía a "un esquema que boy conocemos como 'modelo tutelar', 'filantrópico', 'de la situación irregular', o 'asistencialista', que tenía como punto de partida la consideración del menor como objeto de protección, circunstancia que legitimaba prácticas peno-custodiales y represivas encubiertas" (Beloff, 2004).

En el patronato, la utilización de estigmas tales como "riesgo", "abandono", "fuga del hogar", "niños de la calle y en la calle", entre otros, permitían tipificar una clase de personas que se encontraban "en situación irregular", conformando una identidad social que los aislaba de su entorno. Se pretendía salvar al niño considerándolo peligroso. De ese modo, en lugar de adoptar políticas públicas preventivas de protección integral de la familia y sus hijos, que mejoraran la calidad de sus vidas y las fortalecieran en su función de crianza y educación, se acudía a la "justicia de menores", mediante el alejamiento del niño del binomio familia-escuela, que sólo lograban marginarlo y excluirlo de su vida social.

De allí que se haya dicho que "La utilización del aparato de la administración de justicia en forma sistemática para este estrato poblacional ha estado desde siempre directamente vinculada a la ausencia de políticas públicas de promoción y desarrollo" (Guemureman y Daroqui, 2001). 
Desde el lado opuesto, la doctrina de la "protección integral" implica la consideración de los niños, niñas y adolescentes como sujetos de derechos, es decir, como titulares de todos los derechos contemplados en los instrumentos internacionales y, a la vez, tienen derechos específicos previstos, precisamente, por su condición de personas en etapa de crecimiento.

Este nuevo paradigma implicó no sólo la introducción de garantías a la respuesta estatal al delito de los jóvenes, sino a la posibilidad de discutir la formulación de políticas públicas desde una perspectiva de derechos humanos, teniendo como eje al niño como sujeto de derechos. En efecto, se circunscribe la labor del juez a la resolución de conflictos de naturaleza jurídica, fortalece las garantías procesales y obliga al a la Administración pública a implementar políticas integrales que remuevan los obstáculos que limitan la igualdad y la libertad, impidan o entorpezcan el pleno desarrollo de los niños y su efectiva participación en la comunidad; todo lo cual constituye una condición para el ejercicio pleno de los derechos reconocidos por la Convención sobre los Derechos del Niño.

El sistema requiere la creación de un conjunto de órganos, entidades y servicios de promoción y protección integral de los derechos del niño y el adolescente integrado por órganos administrativos; órganos judiciales y organizaciones de atención a la niñez y la juventud que formulen, coordinen, orienten, supervisen, ejecuten y controlen las políticas, programas y acciones en el ámbito provincial y municipal, incluyendo al sector privado y con amplia participación de la sociedad civil.

Cuando se habla del interés superior del niño, tienen que conjugarse todos los derechos, y el derecho a que se fortalezca su vínculo familiar es contemplado como objeto de protección desde dos directrices: 1) La falta de recursos materiales no constituye causa de separación de los niños y jóvenes de su grupo familiar. 2) El compromiso del Estado debe incluir a esa familia en programas de asistencia, promoción e integración social, garantizando salud, educación y vivienda, mediante la realización de políticas públicas a tales fines. 


\section{Amparo y desamparo}

En causa "Asociación Miguel Bru y otros c/ Provincia de Buenos Aires y otros s/ Amparo", que tramitó en el Juzgado de Primera Instancia en lo Contencioso Administrativo nro. 1 de La Plata, se presentaron tanto los afectados directos -madres de niños y niñas en situación de calle- como organizaciones no gubernamentales (Asociación Civil Miguel Bru, Autoconvocados, Titulares de Cátedra de la Carrera de Trabajo Social de la UNLP), por un lado, y el Ministerio Público Pupilar -Asesoría de Incapaces $\mathrm{N}^{\mathrm{o}} 4$ de La Plata- por el otro; todos reclamando la efectiva y eficiente implementación del nuevo régimen legal de protección de la infancia en la Provincia de Buenos Aries, a fin de promover y proteger los derechos de los niños, niñas y adolescentes que se encuentran en situación de calle en la ciudad de La Plata.

El expediente llegó a superar los 20 cuerpos, en los que se denunció, demostró y evidenció la falta de implementación del SPPN.

Asimismo, el proceso en sí mismo, se erigió como una herramienta de diagnóstico acerca del estado de implementación de la ley 13.298, pues le ha permitido a las autoridades públicas contar con información actualizada acerca de la problemática de la niñez y familia -inexistente al momento de la interposición de la demanda-, pues contiene datos, informes y estadísticas entre otros elementos que se han ido colectando, de la mano de las pruebas realizadas así como de los amicus curiae presentados, que evidenciaron cuáles eran los obstáculos en el diseño, instrumentación, ejecución y control de políticas dirigidas a la niñez

Desde una perspectiva general acerca del funcionamiento de las áreas de niñez, se puso de manifiesto que si bien se habían implementado una serie de programas destinados a dar cumplimiento al mandato legal, los mismos no resultaban suficientes para cubrir de modo efectivo las demandas que afectaban al colectivo de niños, niñas y adolescentes en situación de 
calle en la ciudad de La Plata. Se explicitó como una constante la escasa cantidad de recursos, la precariedad de las intervenciones, la falta de protocolos de actuación y la construcción diaria de estrategias basadas en las convicciones propias de los operadores, evidenciando que las diferentes intervenciones estatales no estaban sustentadas en políticas públicas serias que permitieran un trabajo coherente y sostenido en el tiempo.

En punto al funcionamiento de un "parador" se logró constatar que el establecimiento indicado por las autoridades públicas, no reunía las características adecuadas para brindarles a los niños la contención necesaria, pues si bien las condiciones edilicias eran aceptables para recibir un número aproximado de doce niños, carecía de un equipo técnico y profesional indispensable, ya que la planta, conformada por cuatro asistentes administrativos sin capacitación técnica específica y laa única actividad de recreación disponible era ver televisión.

Con relación a la creación de un Servicio Hospitalario Especializado en salud mental y adicciones, se determinó que si bien la atención de la salud de los niños, niñas y adolescentes de la ciudad de La Plata, se encuentra garantizada -en su etapa aguda- con los servicios de urgencia y de internación del sistema de salud pública, se advirtió la inexistencia de una institución adecuada a la cual puedan ser luego derivados para su rehabilitación o asistencia. Para los casos graves de adicciones que reflejan un marcado deterioro de la salud del niño, el tratamiento para ser eficaz, no puede depender de la voluntad del niño, y en este sentido se acreditó que no existen sistemas que respondan a estas características, con personal auxiliar y profesional suficiente, entrenado, comprometido y sensibilizado con el cuidado integral del niño; y articulación con programas de acompañantes para el egreso y sostenimiento de proyectos inclusivos, con continuidad con sus requerimientos de tratamiento ambulatorio, educación, deporte, recreación, capacitación, etc.

En cuanto a la cantidad de vehículos disponibles para el traslado de niños y niñas en situación de calle se pudo acreditar 
que de los 12.045 vehículos oficiales propiedad del Poder Ejecutivo Provincial, 178 correspondían al Ministerio de Desarrollo Social, pero sin embargo ninguno de ellos había sido destinado para el traslado de niños.

Respecto del funcionamiento de un servicio de atención telefónica destinado a la recepción de denuncias vinculadas a la vulneración de los derechos de niños, niñas y adolescentes, se probó que línea gratuita perteneciente a la Municipalidad funcionaba de manera irregular, y que el Servicio Zonal no contaba con una línea de real y fácil acceso a disposición de toda la comunidad.

Asimismo, se constató que la cantidad de operadores de calle resultaba insuficiente para atender las necesidades del colectivo de niños, niñas y adolescentes en situación de calle en la ciudad de La Plata, situación que se veía agravada por la falta de articulación entre los diferentes organismos que componen el SPDDN.

Tras cuatro años, el 22 de mayo del año 2012 se dictó la sentencia que hizo lugar a la acción de amparo, condenando a la Provincia de Buenos Aires y a la Municipalidad de La Plata a que, dentro del plazo de seis meses procedieran a realizar todas las acciones necesarias para la implementación efectiva del SPPDN en la ciudad de La Plata.

En particular la sentencia establecía que debían: 1) Crear en un ámbito céntrico de la Ciudad, uno o más Paradores, de acuerdo a la demanda del sector, con suficiente infraestructura y personal idóneo para cubrir las necesidades básicas de alimento, higiene, descanso, recreación y contención, de los niños, niñas y adolescentes que requieran esta asistencia, sea en forma espontánea o a requerimiento de quienes pudieran peticionar por ellos, disponible durante las veinticuatro horas del día. Los mismos deberán contar un equipo interdisciplinario integrado, como mínimo, por trabajadores sociales, psicólogos, abogados y médicos especialistas en clínica, pediatría, toxicología y psiquiatría; 2) Crear un Servicio Hospitalario Especializado para la 
atención de la salud de niños con problemas de adicciones $u$ otras afecciones a la salud, que garantice la atención adecuada durante las veinticuatro horas del día, con profesionales idóneos, en particular trabajadores sociales, psicólogos y médicos especialistas en clínica, pediatría, toxicología y psiquiatría, con una capacidad suficiente para atender la demanda del sector, no sólo de aquellos niños institucionalizados, sino también de los que se presenten espontáneamente; 3) Disponer la cantidad de automotores necesaria para efectuar todos los traslados que requieran los niños en situación de vulnerabilidad que se encuentren en la ciudad de La Plata; 4) Implementar un servicio de atención telefónica -amplia y periódicamente difundidodestinado a la recepción de denuncias vinculadas con la vulneración de derechos de los niños, niñas y adolescentes, durante las veinticuatro horas del día, con disposición de operadores que brinden posibilidades concretas de solución de las problemáticas que se planteen; 5) Garantizar la disposición de operadores de calle en cantidad suficiente de acuerdo con las necesidades del colectivo, que comprenda a la división territorial (barrios) de la ciudad de La Plata; 6) Difundir ampliamente en los medios de comunicación masiva de mayor circulación en la ciudad de La Plata, los principios, derechos y garantías de los niños, niñas y adolescentes consagrados en el ordenamiento jurídico, a cuyos efectos, las Administraciones condenadas deberán afectar un porcentaje no inferior al veinticinco por ciento de todas las partidas presupuestarias destinadas a publicidad y/o propaganda oficial para el cumplimiento de la sentencia.

La sentencia fue apelada por la Provincia de Buenos Aires y la Municipalidad de La Plata ante la Cámara de Apelaciones en lo Contencioso Administrativo que el 7 de agosto de 2012 revocó la sentencia en relación a la creación de un servicio hospitalario especializado para la atención de la salud de niños con problemas de adicciones y respecto a la afectación de los recursos económicos para difundir en los medios masivos de comunicación los principios, garantías y derechos de los niños, niñas y adolescentes. 
Contra esa resolución la parte actora con el asesoramiento jurídico del Programa de "Niñez, Derechos Humanos y Políticas Públicas" llevó la causa en el año 2012 a la Suprema Corte de Justicia de la Provincia de Buenos Aires (SCBA), donde se encuentran hasta el día de la fecha.

\section{Justicia desactualizada}

Luego de cuatro años de que el expediente se encontrara radicado en la SCBA sin haberse logrado una solución a la problemática de fondo, en el año 2016 en atención el tiempo transcurrido el tribunal convocó a las distintas partes una audiencia para que se le informe la situación actual de implementación del SPPDN.

En la audiencia, el equipo del Programa de "Niñez, Derechos Humanos y Políticas Públicas" dio cuenta del estado de implementación del SPPDN, de los obstáculos estructurales que debían ser removidos; de cómo se encontraban ahora los niños, niñas y jóvenes que se encontraban en situación de calle al inicio proceso judicial; de otros niños que actualmente se encontraban en situación de calle y de graves episodios vinculados a la niñez y juventud en La Plata.

De aquella exposición, resulta relevante destacar algunos hechos vinculados a las circunstancias que motivaron el amparo, que ocurridos durante el tiempo que la causa estuvo en la órbita de la SCBA esperando el dictado de una sentencia definitiva, demostraron la falta de una efectiva implementación de SPPDN:

En el año 2012 ocurrieron los crímenes de seis niños de 11,14 , y 16 años de edad, bajo distintas circunstancias y modalidades, pero que sin embargo todos tuvieron similares características: a) son muertes violentas; b) se trata de niños y adolescentes en situación de vulnerabilidad atendidos por la Defensoría Oficial del Fuero de la Responsabilidad de Penal Juvenil: c) ocurrieron en las mismas zonas; d) existió algún grado de participación o relación policial en las mismas. 
Las inundaciones ocurridas el 2 de abril de 2013 que afectaron barrios periféricos y el casco urbano de la ciudad de La Plata, pusieron en evidencia la invisibilidad del SPPND. En aquella oportunidad se denunció desde el Programa de "Niñez, Derechos Humanos y Políticas Públicas" la ausencia de funcionarios de niñez durante la catástrofe y la falta total de implementación de un plan de emergencias para los niños y niñas afectados.

El 4 de abril de 2014 el Poder Ejecutivo dictó el Decreto $\mathrm{n}^{\mathrm{o}} 220$ por el cual declaró la emergencia en materia de seguridad pública, destinando cuantiosos recursos económicos a través de la contratación de personal policial, compra directa de equipamiento y patrulleros y la creación de fiscalías especializadas. En aquel contexto, altos funcionarios del ejecutivo realizaron declaraciones públicas instando la necesidad de modificar el Régimen Penal Juvenil, vinculando dogmáticamente niñez y delincuencia. Frente a ello, en el marco del proceso judicial y en calidad de amicus curiae Adolfo Pérez Esquivel -premio Nobel de la Paz- y otras organizaciones sociales vinculadas a la niñez, denunciaron que el aumento del presupuesto de seguridad, frente a la disminución del destinado al área de niñez, junto a las arbitrarias estigmatizaciones que formulaban los funcionarios del poder ejecutivo, constituía una violación al principio de progresividad de en materia de derechos humanos.

En ese mismo año se denunció el desfinanciamiento y la amenaza de traslado del Programa Centro de Tratamiento Integral (Ce'TAI), destinado a abordar problemáticas de niños, niñas y jóvenes como trastornos de socialización, conductas disruptivas, exclusiones de instituciones nucleares, entre otras. El desfinanciamiento al que se vio expuesto, sumado al traslado a un lugar que no reunía las condiciones mínimas de habitabilidad, constituía una medida regresiva respecto de la política de salud mental destinada a los niños y niñas que pudo ser evitada miente medida autosatisfactiva.

Desde mediados de julio de 2015 el diario "El Día” publicó más de 20 notas en torno a un grupo de niños, identificándolos 
como la "Banda de los nenes" que "se habría especializado en delinquir". De la lectura de los reportes, surge la estigmatización de estos niños, la invisibilización de la biografía de cada uno de ellas, y la ausencia de las políticas de promoción protección integral de sus derechos, resultando que el Estado se vincula con estos niños a través de la policía que sistemáticamente los aprehende y los traslada a alguna comisaría.

Durante el 2016, un grupo de aproximadamente 20 niños de entre 5 y 15 años, en situación de calle, incluyeron en su tránsito urbano el recorrido de distintas unidades académicas de la UNLP con el objetivo de obtener dinero, comida y pasar tiempo de recreación. En esos recorridos se han generado algunas situaciones conflictivas que terminaron en la expulsión y la prohibición de ingresos de los niños a esos ámbitos, sin intervención del SPPND.

\section{Conclusión}

Han pasado más de 10 años desde aquella noche en que se pretendió "limpiar la plaza". Desde entonces, aquellos niños y aquellas niñas, ya no lo son. Algunos de ellos han traspasado la frontera de la muerte, a manos del gatillo fácil. Muchos están encerrados en la cárcel. Varios de ellos y ellas han sufrido el abuso, la explotación sexual y padecido la violencia en sus múltiples formas. El Estado los abandonó, los castigo, los penalizó, los golpeó, los hostigo, los encerró y les puso muerte (la propia y ajena) en sus manos.

La prolongación del proceso y la demora en el dictado de una sentencia por parte de la SCBA incide de manera relevante y cierta en la ausencia de acciones positivas por parte del Ejecutivo en implementar eficazmente el SPPDN, generando daños de carácter irreversible para algunos niños, niñas y adolescentes.

La realidad apremia y castiga a los más pequeños, demostrando la actualidad del reclamo y la vigencia de que el Estado aporte soluciones a una cuestión que es compleja debido a que su resolución excede el campo de lo jurídico e involucra la 
necesidad de acciones concretas tendientes a la efectiva implementación de un cambio de paradigma que se proyecte en el campo cultural de la población, propiciando cambios que incidan efectivamente en la realidad cotidiana de los niños, niñas y jóvenes de la Provincia de Buenos Aires.-

La SCBA tiene la oportunidad histórica de dictar una sentencia ejemplar que active los resortes burocráticos para que el Estado, los medios de comunicación y la sociedad en su conjunto seamos capaces de torcer esta mirada triste sobre la niñez, y que de una vez y para siempre podamos ofrecerles a todos los niños, niñas y jóvenes una opción de vida que los enamore y los conquiste, reconociendo sus deseos y necesidades.

El Poder Judicial puede y debe inaugurar un proceso que conduzca a saldar la terrible deuda que tenemos los adultos, abriendo nuevos caminos que permitan construir una comunidad respetuosa de los derechos de los niños, niñas y jóvenes.

\section{Bibliografía}

Belauzarán, Lucia et al (2015) Niñez y Derechos humanos. Herramientas para un abordaje integral. Editorial UNLP, La Plata.

Beloff, Mary (2004). Los derechos del niño en el sistema interamericano, Editores del Puerto.

Gargarela, Roberto et al (2005). "El derecho de resistencia en situaciones de carencia extrema" en $E l$ derecho a resistir el derecho. Miño y Dávila Editores, Buenos Aires.

Guemureman, Silvia y Daroqui, Alcira (2001). La niñez ajusticiada, Editores Del Puerto, Buenos Aires.

Naciones Unidas, Comité de los Derechos del Niño, Observaciones Finales: Argentina, CRC/C/15.Add.187, del 9-X-2002. 